\title{
Kinesin-12, a Mitotic Microtubule-Associated Motor Protein, Impacts Axonal Growth, Navigation, and Branching
}

\author{
Mei Liu, ${ }^{1,2}$ Vidya C. Nadar, ${ }^{1}$ Frank Kozielski, ${ }^{3}$ Marta Kozlowska, ${ }^{3}$ Wenqian Yu, ${ }^{1 *}$ and Peter W. Baas ${ }^{1 *}$ \\ ${ }^{1}$ Department of Neurobiology and Anatomy, Drexel University College of Medicine, Philadelphia, Pennsylvania 19129, ${ }^{2}$ Jiangsu Key Laboratory \\ of Neuroregeneration, Nantong University, Nantong, Jiangsu 226001, China, and ${ }^{3}$ The Beatson Institute for Cancer Research, Glasgow G61 1BD, \\ United Kingdom
}

\begin{abstract}
Kinesin-12 (also called Kif15) is a mitotic motor protein that continues to be expressed in developing neurons. Depletion of kinesin-12 causes axons to grow faster, more than doubles the frequency of microtubule transport in both directions in the axon, prevents growth cones from turning properly, and enhances the invasion of microtubules into filopodia. These results are remarkably similar to those obtained in previous studies in which neurons were depleted of kinesin-5 (also called Eg5 or Kif11), another mitotic motor protein that continues to be expressed in developing neurons. However, there are also notable differences in the phenotypes obtained with depleting each of these motors. Depleting kinesin-12 decreases axonal branching and growth cone size, whereas inhibiting kinesin-5 increases these parameters. In addition, depleting kinesin-12 diminishes the appearance of growth-cone-like waves along the length of the axon, an effect not observed with depletion of kinesin-5. Finally, depletion of kinesin-12 abolishes the "waggling" behavior of microtubules that occurs as they assemble along actin bundles within filopodia, whereas inhibition of kinesin-5 does not. Interestingly, and perhaps relevant to these differences in phenotype, in biochemical studies, kinesin-12 coimmunoprecipitates with actin but kinesin-5 does not. Collectively, these findings support a scenario whereby kinesin-12 shares functions with kinesin-5 related to microtubule-microtubule interactions, but kinesin-12 has other functions not shared by kinesin- 5 that are related to the ability of kinesin-12 to interact with actin.
\end{abstract}

\section{Introduction}

Decades ago, the microtubule array of the axon was viewed as a coherent slow-moving mass of polymers, transported throughout the life of the neuron, from the cell body toward the tip of the axon (Lasek, 1986). Today we know that only a small portion of the microtubules within the array undergoes transport at any given moment, and when this happens, the transport occurs rapidly, at the rate of known molecular motor proteins (Wang and Brown, 2002). Interestingly, only very short microtubules undergo transport, and the transport occurs in both directions. We have proposed that a variety of molecular motors generate forces on axonal microtubules, regardless of their length (Baas et al., 2006). In the case of the longer microtubules, these forces do not move the microtubules, but nevertheless are critical for determining whether the axon grows or retracts, and for regulating the proper turning of the growth cone. This scenario is reminiscent of the mitotic spindle, wherein even during phases of little movement, the microtubules are subjected to forces generated by a host of motor proteins (Walczak and Heald, 2008). Shifts in the

\footnotetext{
Received July 18, 2010; revised Aug. 15, 2010; accepted Sept. 12, 2010.

This work was supported by grants to P.W.B. from the National Institutes of Health (NIH) and the Christopher and Dana Reeve Foundation, and a grant to W.Y. from the Craig H. Nielsen Foundation. For helpful discussions, we thank Drs. Timothy Mitchison of Harvard University, Kenneth Myers of NIH, Liang Qiang of Columbia University, and Miche Kress of the CNRS

*W.Y. and P.W.B. are co-senior authors of this work.

Correspondence should be addressed to Peter W. Baas, Department of Neurobiology and Anatomy, Drexel University College of Medicine, 2900 Queen Lane, Philadelphia, PA 19129. E-mail: pbaas@drexelmed.edu.

DOI:10.1523/JNEUROSCI.3739-10.2010

Copyright $\odot 2010$ the authors $\quad 0270-6474 / 10 / 3014896-11 \$ 15.00 / 0$
}

balance of the motor-driven forces are instrumental for the progression of mitosis, and we posit that the same is true of various aspects of axonal morphogenesis.

In studies on cultured neurons, we have shown that inhibiting or depleting cytoplasmic dynein causes a reduction in the frequency of microtubule transport in the axon in the anterograde direction but not in the retrograde direction (He et al., 2005; Ahmad et al., 2006). The axon becomes more prone to retraction and the growth cones tend to stop at substrate borders rather than turn (Ahmad et al., 2000; Myers et al., 2006). When we deplete or inhibit kinesin-5, the frequency of short microtubule transport almost doubles in both directions, the axon grows faster and becomes less prone to retraction (Haque et al., 2004; Myers and Baas, 2007), and growth cones ignore cues that would otherwise cause them to turn (Nadar et al., 2008).

Kinesin-5, a homotetrameric motor also called Eg5 or Kif11, is best known for its role in mitosis, specifically in the separation of the half-spindles (Kashina et al., 1996; Scholey, 2009; Ferenz et al., 2010). Kinesin-12, a bipolar motor also called Kif15, is another mitotic motor that continues to be expressed in developing neurons (Buster et al., 2003). Although not as well studied as kinesin-5, kinesin-12 is believed to be important for maintaining half-spindle separation by opposing forces generated by other motors (Wittmann et al., 1998; Rogers et al., 2000). In fibroblasts, kinesin-12 alternately colocalizes with either microtubules or actin filaments, depending on the phase of the cell cycle (Buster et al., 2003). Given that many aspects of axonal morphogenesis involve the coordinated interplay between these two cytoskeletal elements, the potential ability of kinesin-12 to interact with both 
A

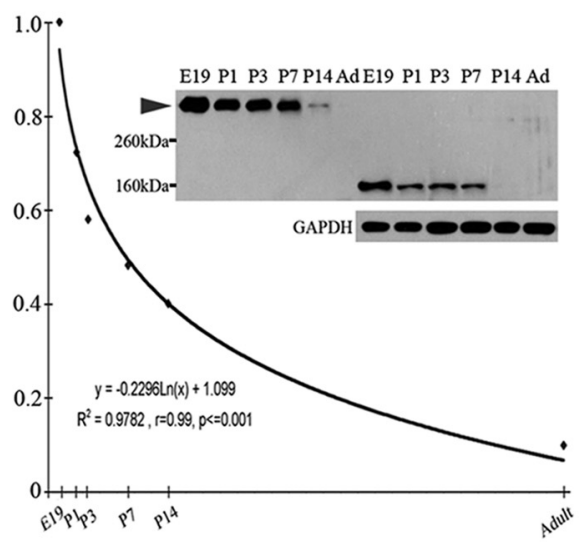

B

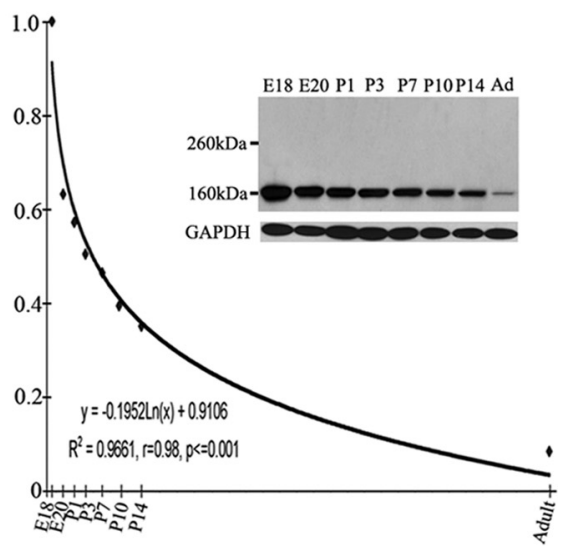

Figure 1. Kinesin-12 expression in the nervous system during development. $\boldsymbol{A}$, The plot shows nonlinear correlation analyses of the levels of kinesin-12 in rat cortex during different development stages of E19, P1, P3, P7, P14, and adult: $(y=-0.2296 \ln (x)+1.099, r=0.99, p \leq 0.001)$. The inset is a representative Western blot of the levels of kinesin-12 protein. The upper bands (arrowhead) at $\sim 320 \mathrm{kDa}$ indicate the dimeric kinesin- 12 protein from nonreducing conditions, while the lower bands at $\sim 160 \mathrm{kDa}$ are monomeric kinesin- 12 protein from reducing conditions. GAPDH was used as internal control. $\boldsymbol{B}$, The plot shows nonlinear correlation analyses of the levels of kinesin- 12 in rat sympathetic ganglia during different development stages of $\mathrm{E} 18, \mathrm{E} 20, \mathrm{P} 1, \mathrm{P} 3, \mathrm{P} 7, \mathrm{P} 10, \mathrm{P} 14$, and adult $(y=-0.1952 \ln (x)+0.9016, r=0.98$, $p \leq 0.001$ ). The inset is a representative Western blot of the levels of kinesin- 12 protein in rat sympathetic ganglia. The studies on rat sympathetic ganglia were conducted only under reducing conditions, revealing the $160 \mathrm{kDa}$ monomeric form.

has compelling functional implications. Here, we conducted studies on the effects of kinesin-12 depletion on microtubule behaviors in the axon as well as axonal growth, navigation, and branching.

\section{Materials and Methods}

Generation of kinesin-5 and kinesin-12 antibodies. The cDNAs encoding residues 801-991 of rKinesin-5 (GenBank No. XM_001060913) and 1146-1385 of rKinesin-12 (GenBank No. NM_181635.2) were obtained with HF PCR using rat fetal brain cDNA (Clontech) as a template. The primers (rKinesin-5-tail-sense: $5^{\prime}$-ccg gaattc atg tgt ggt tca ctc agc agc aac- $3^{\prime}$ and antisense: $5^{\prime}-\mathrm{ccg}$ ctcgag tca act tac gag ttc ttc agt act ctg ctc- $3^{\prime}$; rKinesin-12-tail-sense: $5^{\prime}$-ccg gaattc atg caa act cat ttg gca aaa ctc ttg- $3^{\prime}$ and antisense: $5^{\prime}-\mathrm{ccg}$ ctcgag tca ttc ttt ttt cct ttc ttt caa aaa tac- $3^{\prime}$ ) were synthesized by Integrated DNA Technologies. The cDNAs were restriction digested with EcoR I and XhoI (NEB) and then cloned into pET-32a (Novagen). The sequences of the clones were confirmed by DNA sequencing performed by GENEWIZ. These constructs were used for protein expression in Escherichia coli BL21 (DE3), and the corresponding His-rKinesin-5 tail and His-rKinesin-12 tail fusion proteins were purified using standard procedures. The rKinesin- 12 tail antisera and rKinesin- 5 tail antisera were generated by Cocalico Biological by immunizing at least two different rabbits in each case. The sera were affinity purified using IgG covalently bound to a HiTrap column (GE Healthcare Life Sciences). The affinity-purified antibodies were used at $1.0 \mu \mathrm{g} / \mathrm{ml}$. The new rKinesin-12 antibody was used for Western blotting and immunoprecipitation, and the new rKinesin-5 antibody was used for Western blotting. For immunostaining of kinesin-12, we used our earlier antibody to kinesin-12 generated against the stalk domain (Buster et al., 2003), and for immunoprecipitation of kinesin-5, we used a commercially available kinesin-5 antibody (Abcam, ab37009).

Western blotting. Total protein from rat neuronal tissues (cerebral cortex, sympathetic ganglia) were extracted at different stages of development [embryonic day 19 (E19), postnatal day 1 (P1), P3, P7, P14, and adult from cortex; E18, E20, P1, P3, P7, P10, P14, and adult from sympathetic ganglia] using CelLytic microtubule Cell Lysis Reagent (Sigma). The total protein was quantified using the BCA Protein Assay Kit (Pierce) and then adjusted to equal concentration and stored at $-80^{\circ} \mathrm{C}$ in small aliquots. In some studies, protein samples were treated under nonreducing conditions as described previously (Kage et al., 2005). The Bio-Rad Mini-PROTEAN Tetra Cell system was used for electrophoretic separa- tion of the protein samples as per the instructions from Bio-Rad Laboratories. Fifteen micrograms of protein samples were loaded in each lane and resolved using 7.5\% SDS-PAGE for kinesin- 12 and kinesin-5 and 10\% SDS-PAGE for glyceraldehyde-3-phosphate dehydrogenase (GAPDH) (internal control). The gel was transferred onto nitrocellulose membranes [Bio-Rad, catalog number (Cat.) 162-0232], and the blots were blocked and then probed with rabbit anti-kinesin-12 tail polyclonal antibody at $4^{\circ} \mathrm{C}$ overnight. After washing, the blots were incubated with peroxidase-conjugated anti-rabbit IgG (Jackson ImmunoResearch). Membrane-bound peroxidase was visualized on Pierce* CL-XPosure Film after enhancement with ECL Western Blotting Substrate (Pierce). Mouse monoclonal anti-GAPDH (Sigma) and the corresponding peroxidase-conjugated antimouse IgG (Jackson ImmunoResearch) were used.

Sympathetic and cortical neuronal cells culture and siRNA transfection. Sympathetic and cortical neuronal cultures were prepared and transfected using the Nucleofector device (Amaxa) as previously described (He et al., 2005). The kinesin-12 siRNA [ONTARGETplus SMARTpool siRNA (Dharmacon, Cat. L097130-01)] or nonspecific control siRNA (Ambion) for transfection were used at a concentration of 10 $\mu \mathrm{mol} / \mathrm{L}$. After transfection, neurons were plated on $35 \mathrm{~mm}$ culture dishes (Corning) that had been coated with $0.1 \mathrm{mg} / \mathrm{ml}$ poly-D-lysine (Sigma, Cat. P0899) for sympathetic neurons and $1 \mathrm{mg} / \mathrm{ml}$ poly-L-lysine (Sigma, Cat. P2636) for cortical neurons. As previously reported, introduction of siRNA with this procedure is generally successful in virtually all of the neurons, and effective in diminishing protein levels as assessed by immunostaining (He et al., 2005). The replating procedure results in robust healthy cultures in which phenotypes can be evaluated after substantial reduction in the levels of the targeted protein (for more discussion, see Tint et al., 2009). On this basis, neurons were selected randomly in the case of both control and experimental cultures for the various morphological and functional assays.

Immunocytochemistry. Neuronal cultures were fixed at different times as described previously (Myers and Baas, 2007; Nadar et al., 2008). Cy3conjugated mouse monoclonal anti- $\beta$-tubulin Ab (1:800; Sigma Cat. C4585) was used for immunostaining of tubulin, and Alexa Fluor 488 phalloidin (1:40; Molecular Probe, Cat. A12379) was used to reveal filamentous actin (F-actin) in double-staining experiments. For triple staining of tubulin, F-actin, and cofilin, cultures were incubated with mouse monoclonal anti- $\beta$-tubulin (1:400; Sigma, Cat. T4026) and rabbit polyclonal anti-cofilin antibody (1:2000; Abcam, Cat. ab42824) at $4^{\circ} \mathrm{C}$ for $14-16 \mathrm{~h}$ and then incubated in secondary antibodies (Cy5-conjugated goat anti-mouse IgG at 1:200, Cy3-conjugated goat anti-rabbit IgG, 1:800, Jackson ImmunoResearch; and Alexa Fluor 488 phalloidin, 1:40) for $1 \mathrm{~h}$ at $37^{\circ} \mathrm{C}$. Fluorescence images were acquired as described previously (Hasaka et al., 2004). In one set of experiments, RFL-6 fibroblasts were immunostained for kinesin-12 as previously described (Buster et al., 2003). Immunostaining for the border assay is described below.

Border assay. We used a modification of the border assay used in previous studies to evaluate growth cone turning (Turney and Bridgman, 2005; Nadar et al., 2008). Sympathetic neuronal cultures were prepared and transfected with control siRNA and kinesin-12 siRNA and plated on laminin-coated $(100 \mu \mathrm{g} / \mathrm{ml})$ plastic dishes for $2 \mathrm{~d}$. The coverslips for the border were prepared by adding $50 \mu \mathrm{l}$ of laminin $(25 \mu \mathrm{g} / \mathrm{ml})$ in the center of poly-DL-lysine-coated (Sigma, Cat. P9011;0.1 mg/ml in borate buffer) coverslips and then incubated at $37^{\circ} \mathrm{C}$ for $3 \mathrm{~h}$. Laminin was removed and the coverslips were rinsed with $50 \mu \mathrm{l}$ of $\mathrm{ddH}_{2} \mathrm{O}$ three times before replating the cells. Each $45 \mu \mathrm{l}$ of the cultured neurons treated with control or kinesin-12 siRNA were replated to the central region coated with lami- 

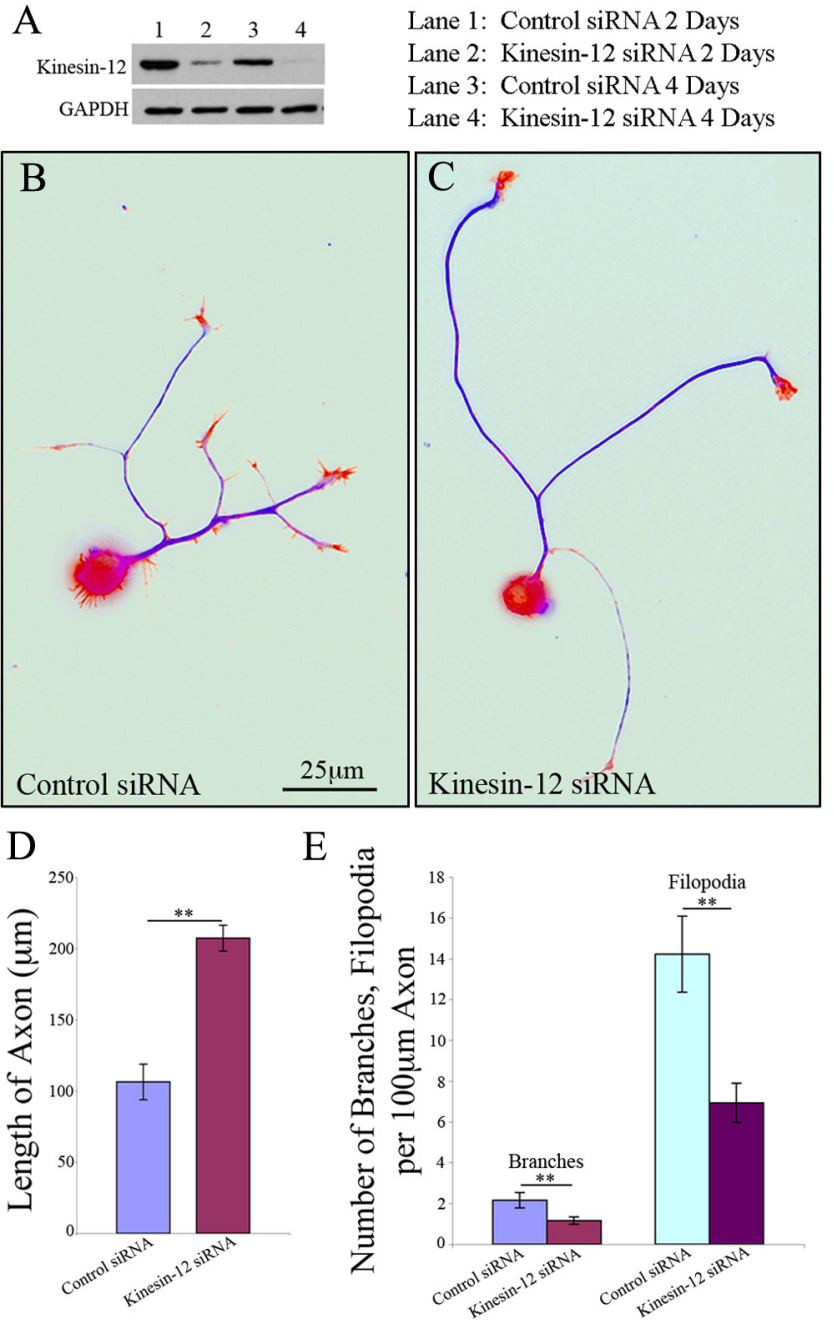

Figure 2. Effects of kinesin-12 depletion on the morphology of primary neurons from rat sympathetic ganglia. $\boldsymbol{A}$, Western blot showing levels of kinesin-12 in cultured sympathetic neurons transfected with control siRNA and kinesin-12 siRNA for 2 and $4 \mathrm{~d}$. The bottom panel shows the blot for GAPDH, which was used as a loading control. $B, C$, Representative pseudocolored fluorescence images of neurons transfected with control siRNA $(\boldsymbol{B})$ and kinesin-12 siRNA $(\boldsymbol{C})$, and then fluorescently stained for tubulin (blue) and F-actin (red). Scale bar, $25 \mu \mathrm{m}$. D, Quantification of axonal length in rat sympathetic neurons revealed significantly longer axons in neurons depleted of kinesin-12 compared to neurons transfected with control siRNA. Data are represented as mean $\pm \mathrm{SE}$, control siRNA $=106.3 \pm 12.5 \mu \mathrm{m}, n=36$; kinesin- 12 siRNA $=$ $207.4 \pm 9.0 \mu \mathrm{m} ; n=41,{ }^{* *} p<0.001$. $\boldsymbol{E}$, Quantification of $100 \mu \mathrm{m}$ length of axons in rat sympathetic neurons shows significantly fewer branches and filopodia in neurons transfected with kinesin-12 siRNA compared to neurons transfected with control siRNA. Data are represented as mean $\pm \mathrm{SE}$; axonal branches, control siRNA $=2.2 \pm 0.4 / 100 \mu \mathrm{m}$, kinesin-12 siRNA $=1.2 \pm 0.2 / 100 \mu \mathrm{m}$; actin filopodia, control siRNA $=14.2 \pm 1.9 / 100 \mu \mathrm{m}$, kinesin-12 siRNA $=6.9 \pm 0.9 / 100 \mu \mathrm{m} ;{ }^{*} p<0.05,{ }^{* *} p<0.01$.

nin. After $1 \mathrm{~h}, 1.5 \mathrm{ml}$ of the cell culture medium was added to each dish. The neurons were allowed to grow axons until most of their growth cones reached the border and either crossed or turned, which typically took 24-48 $h$. The cultures were then fixed and fluorescently stained with mouse monoclonal anti- $\beta 3$ tubulin antibody (1:600; Covance) and rabbit anti-laminin antibody (1:800; Sigma). Fluorescence images were acquired and quantified using Axiovision 4.6 as in our previous work (Nadar et al., 2008).

Microtubule transport assay. The microtubule transport assay was performed as described previously (Hasaka et al., 2004). The neurons transfected with either control siRNA or kinesin-12 siRNA together with EGFP-tubulin were replated on poly-D-lysine-coated coverslips after $2 \mathrm{~d}$ as described above. The axons of EGFP-tubulin-transfected neurons were photobleached for $300 \mathrm{~ms}$ using the Mosaic System (Photonics). Time-lapse images were taken at 200-300 ms exposure at $2 \mathrm{~s}$ intervals for

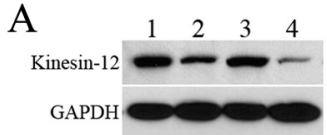

Lane 1: Control siRNA 1 Day

Lane 2: Kinesin-12 siRNA 1 Day

Lane 3: Control siRNA 3 Days

Lane 4: Kinesin-12 siRNA 3 Days

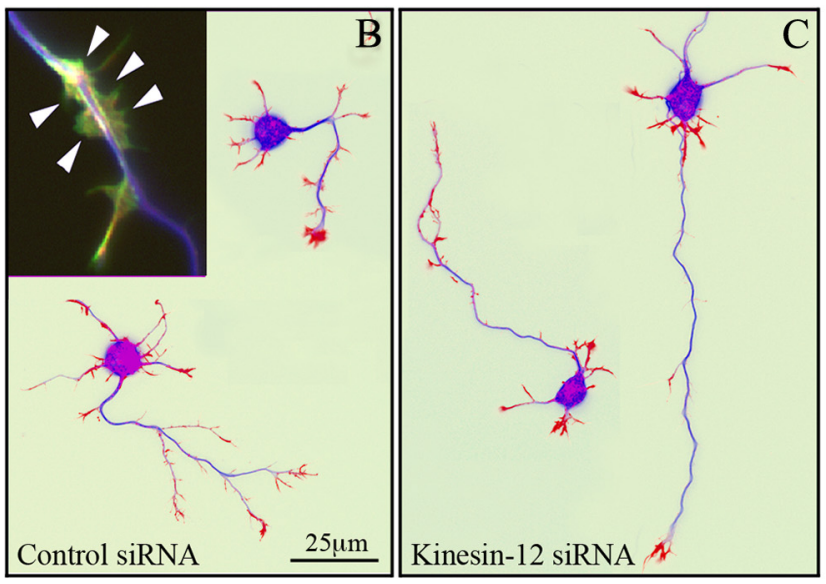

$\mathrm{D}$

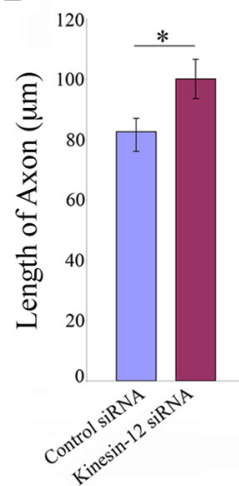

$\mathrm{E} \approx$

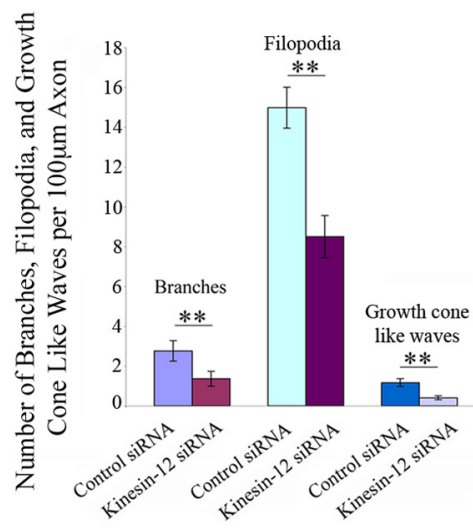

Figure 3. Effects of depletion of kinesin-12 on the morphology of primary rat cortical neurons. $\boldsymbol{A}$, Representative Western blot showing levels of kinesin-12 in cultured cortical neurons transfected with control siRNA or kinesin-12 siRNA for 1 and $3 \mathrm{~d}$. GAPDH was used as a loading control. $\boldsymbol{B}, \boldsymbol{C}$, Representative pseudocolored fluorescence images of rat cortical neurons fluorescently stained for tubulin (blue) and F-actin (red) in neurons transfected with control siRNA (B) or kinesin-12 siRNA ( $\boldsymbol{C}$. The inset in $\boldsymbol{B}$ shows a representative fluorescence image of a growthcone-like wave indicated by arrowheads along the axon (tubulin: blue; F-actin: green; cofilin: red). Cofilin was used as a marker for growth-cone-like waves (see Flynn et al., 2009). Scale bar, $25 \mu \mathrm{m}$. D, Quantification of axonal length in rat cortical neurons revealed significantly longer axons in neurons transfected with kinesin- 12 siRNA as compared to axons of neurons transfected with control siRNA. Data are represented as mean $\pm \mathrm{SE}$; control siRNA $=82.6 \pm 4.4$ $\mu \mathrm{m}, n=45$; kinesin- 12 siRNA $=100.1 \pm 6.5 \mu \mathrm{m}, n=47$; $^{*} p<0.05$. $\boldsymbol{E}$, Quantification in rat cortical neurons showed significantly fewer collateral branches, filopodia, and growth-conelike waves in neurons transfected with kinesin-12 siRNA than in neurons transfected with control siRNA. Data are represented as mean $\pm \mathrm{SE}$; axonal branches, control siRNA $=4.9 \pm$ $0.5 / 100 \mu \mathrm{m}$, kinesin-12 siRNA $=2.5 \pm 0.4 / 100 \mu \mathrm{m}$; actin filopodia, control siRNA $=15.0 \pm$ $1.03 / 100 \mu \mathrm{m}$, kinesin-12 siRNA $=8.5 \pm 1.1 / 100 \mu \mathrm{m}$; actin- and cofilin-positive staining, control siRNA $=1.2 \pm 0.2 / 100 \mu \mathrm{m}$, kinesin-12 siRNA $=0.4 \pm 0.1 / 100 \mu \mathrm{m} .{ }^{* *} p<0.01$.

each axon for $5 \mathrm{~min}$. Transport analyses included all microtubules observed to move continuously through the photobleached region during the imaging period. Transport frequencies were calculated for anterograde or retrograde direction by dividing the total number of movements by the total imaging time for individual movies.

Cloning, expression, purification of kinesin-5 and kinesin-12 motor domain, and studies on potential drug inhibition of kinesin-5 and kinesin-12 ATPase activities. Human kinesin-5 (Eg5 $\left.{ }_{1-368}\right)$ construct was prepared as previously described (Kaan et al., 2009). The cDNA coding for the human kinesin-12 motor domain (residues 43-399, named Kif15 ${ }_{43-399}$, from Genescript) was excised from the pUC57 vector and subsequently ligated into the expression vector pET-M20mod, and sequenced. The 


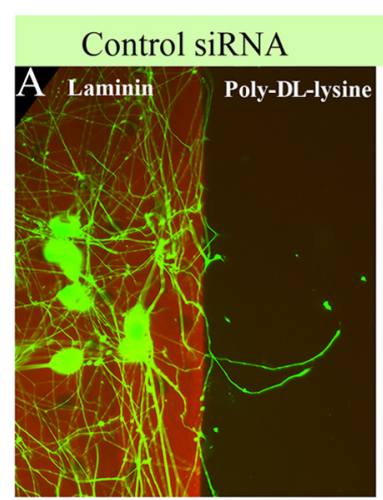

Kinesin-12 siRNA

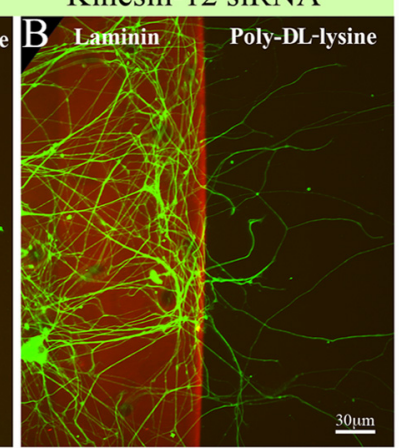

$\mathrm{C}$

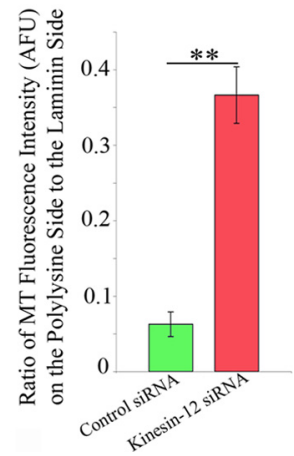

Figure 4. Depletion of kinesin-12 from sympathetic neurons inhibits growth cone turning. $A, B$, Representative fluorescence images of neurons subjected to the border assay (see Materials and Methods). The laminin coating on the coverslip is revealed by immunostaining for laminin shown in red, and the neurons and their axons are visualized by immunostaining for $\beta 3$-tubulin (green). $\boldsymbol{A}$, Axons of neurons transfected with control siRNA turn when the growth cones reach the laminin/polylysine border. $\boldsymbol{B}$, Axons of neurons transfected with kinesin-12 siRNA do not turn at the laminin/polylysine border, but instead cross the border. Scale bar, $30 \mu \mathrm{m}$. C, Quantification of the ratio of microtubule fluorescence on the polylysine side of the border (axons that crossed the border) and the laminin side of the border (axons that did not cross the border) in neurons depleted of kinesin- 12 siRNA ( $0.4 \pm$ $0.04)$ and control neurons $(0.06 \pm 0.02)$. Data are represented as mean $\pm \mathrm{SE}^{* * *} p<0.001$.

kinesin-12 expression construct was transformed into Codon + E. coli cells. The expression and purification of kinesin-12 motor protein was performed as follows: Cells were lysed by sonication using eight cycles of $8 \mathrm{~s}$ of sonication, and subsequently centrifuged at 20,000 rpm for $1 \mathrm{~h}$ at $4^{\circ} \mathrm{C}$. The supernatant $(\sim 100 \mathrm{ml})$ was applied to a $1 \mathrm{ml}$ Histrap FF NiNTA column previously equilibrated in buffer A (50 mM PIPES, pH 7.0, $250 \mathrm{~mm} \mathrm{NaCl}, 10 \mathrm{~mm}$ imidazole, $2 \mathrm{~mm} \mathrm{MgCl}_{2}$ ). The column was washed with $60 \mathrm{ml}$ of buffer B (50 mM PIPES, pH 7.0, $250 \mathrm{~mm} \mathrm{NaCl}, 40 \mathrm{~mm}$ imidazole, $2 \mathrm{mM} \mathrm{MgCl}_{2}$ ), and kinesin-12 was eluted with a gradient from 40 to $500 \mathrm{~mm}$ imidazole. The protein-containing fractions were pooled and applied to a 16/60 desalting column (GE Healthcare) with buffer A (in the absence of any imidazole). The protein was incubated with $3 \mathrm{~mm}$ DTT and $0.05 \mathrm{mg}$ of TEV/ $1 \mathrm{mg}$ of kinesin- 12 overnight at $4^{\circ} \mathrm{C}$ and subsequently passed over an additional desalting column using the same conditions. The protein was pooled and applied to a second $1 \mathrm{ml} \mathrm{Ni-NTA}$ column. Kinesin-12 elutes in the flow through, whereas noncleaved Trxtagged kinesin-12, his-tagged TEV, and contaminations remain bound to the resin. The protein was concentrated to $2.5 \mathrm{ml}$ and applied to a Superose S70 gel filtration column in buffer A supplemented with $1 \mathrm{~mm}$ DTT. After elution from the column kinesin-12 was concentrated to 2 $\mathrm{mg} / \mathrm{ml}$, supplemented with $1 \mathrm{~mm} \mathrm{Mg}{ }^{2+}$ ATP and used for ATPase activity assays without freezing. The inhibition of the basal and microtubulestimulated ATPase activity of kinesin- 5 and kinesin- 12 by monastrol [or $S$-trityl-L-cysteine (STLC)] was measured as previously described (DeBonis et al., 2008). All data were measured at least in triplicate and are reported as means \pm SD.

EGFP-EB3 imaging in growth cones. EB3 is a microtubule end-binding protein that associates with the plus-end of the microtubule during bouts of assembly, and hence EGFP-EB3 appears as fluorescent "comets" at the plus ends of the assembling microtubules (Stepanova et al., 2003). Ectopic expression of EGFP-EB3 has proven to be a convenient approach for visualizing microtubule assembly events in living neurons. Rat neurons from the superior cervical ganglia were prepared and transfected with either control siRNA or kinesin-12 siRNA together with $10 \mu \mathrm{g}$ of EGFP-EB3 to visualize the EB3 comets, as described previously. After $48 \mathrm{~h}$, neurons were replated onto poly-D-lysine-coated glass-bottomed dishes. After $8-12 \mathrm{~h}$, laminin $(5 \mu \mathrm{g} / \mathrm{ml})$ was added to the medium. Images of the growth cones were obtained every second for $3 \mathrm{~min}$ at an exposure time of $600 \mathrm{~ms}$ and $1 \times 1$ binning using Axiovision 4.6. The images were quantified as described previously for the number of comets that entered the filopodia, and the velocity of the comets that enter in axonal shaft and filopodia (Nadar et al., 2008). In some studies, we treated the cultures with $100 \mu \mathrm{M}$ monastrol (in $0.1 \%$ DMSO) and $0.1 \%$
DMSO was used as control. The comet trajectories were traced in these experiments using MetaMorph software (Universal Imaging).

Coimmunoprecipitation. A commercially available Co-Immunoprecipitation Kit (Pierce, Cat. 23600) was used to investigate a potential interaction between kinesin-12 and actin. Briefly, RLF-6 cells were lysed in NP-40 Cell Lysis Buffer (containing Protease Inhibitor Cocktail, Pierce). Our new anti-rKinesin-12 tail antibody and a commercially available anti-actin antibody (Abcam, Cat. 3280) were immobilized in Antibody Coupling Resin and Control Resin, respectively, supplied in the kit. Coimmunoprecipitation and elution of coimmunoprecipitation complex, preparation of samples for SDS-PAGE were performed as per the instructions in the kit, respectively. Western blotting was used to determine the presence of actin and kinesin-12 in the coimmunoprecipitation complex. The kinesin-12 antibody and anti-actin antibody (Sigma, Cat. A2066) were used for immunoblotting. A commercially available kinesin-5 antibody (Abcam, ab37009) was used for coimmunoprecipitation assay of kinesin-5 and actin, and our antirKinesin-5 tail antibody was used to detect kinesin- 5 on the Western Blots.

Statistical analysis and image processing. All data analyses, statistical comparisons, and graphs were generated using Excel (Microsoft) or Kaleidagraph. Data represent mean \pm SEM of three or four separate experiments for each assay, and comparison was performed using a two-tailed $t$ test or univariate ANOVA. For all statistical analysis, the mean difference was considered to be significant at the 0.05 level. The final figure processing was performed with Photoshop 9.0 (Adobe).

\section{Results}

\section{Developmental expression of kinesin-12}

In a first set of studies, we wished to ascertain the pattern of expression of kinesin-12 in the CNS and peripheral nervous system (PNS). For this, we conducted quantitative Western blotting on homogenized tissues from rat cortex (CNS) and superior cervical ganglia (PNS) (Fig. 1). The predicted molecular weight of kinesin-12 is $\sim 160 \mathrm{kDa}$. Our new polyclonal antibody against the tail domain of rat kinesin-12 produced a single band on Western blots that corresponded precisely to the predicted molecular weight. The band was diminished in cells treated with siRNA for kinesin-12 (Fig. 2A), confirming the identity of this band as kinesin-12. As shown in Figure 1, $A$ and $B$, the levels of kinesin-12 are high in both cortex and ganglia at embryonic stages but then progressively diminish as development ensues. Beyond P14, the levels are barely detectable. When the samples were treated under nonreducing conditions, the band appeared at twice the molecular weight, which is consistent with the prediction that kinesin-12 exists as a dimer in the cytoplasm of these tissues (Fig. $1 A$, arrowhead).

\section{Depletion of kinesin-12 impacts PNS neuronal morphology}

To ascertain the morphological impact of depleting kinesin-12, we used the same procedure we used in the past for siRNA-based depletion of motor proteins from cultured neurons (Myers and Baas, 2007; Nadar et al., 2008). Specifically, a pool of four different siRNA sequences specific to rat kinesin-12 was transfected by electroporation into the neurons, which were then plated densely on $0.1 \mathrm{mg} / \mathrm{ml}$ poly-D-lysine-coated plastic dishes for $2 \mathrm{~d}$. During this time period, $>90 \%$ of the kinesin- 12 protein was depleted [as assessed by Western blotting (Fig. 2A)]. At this point, the cells 
A

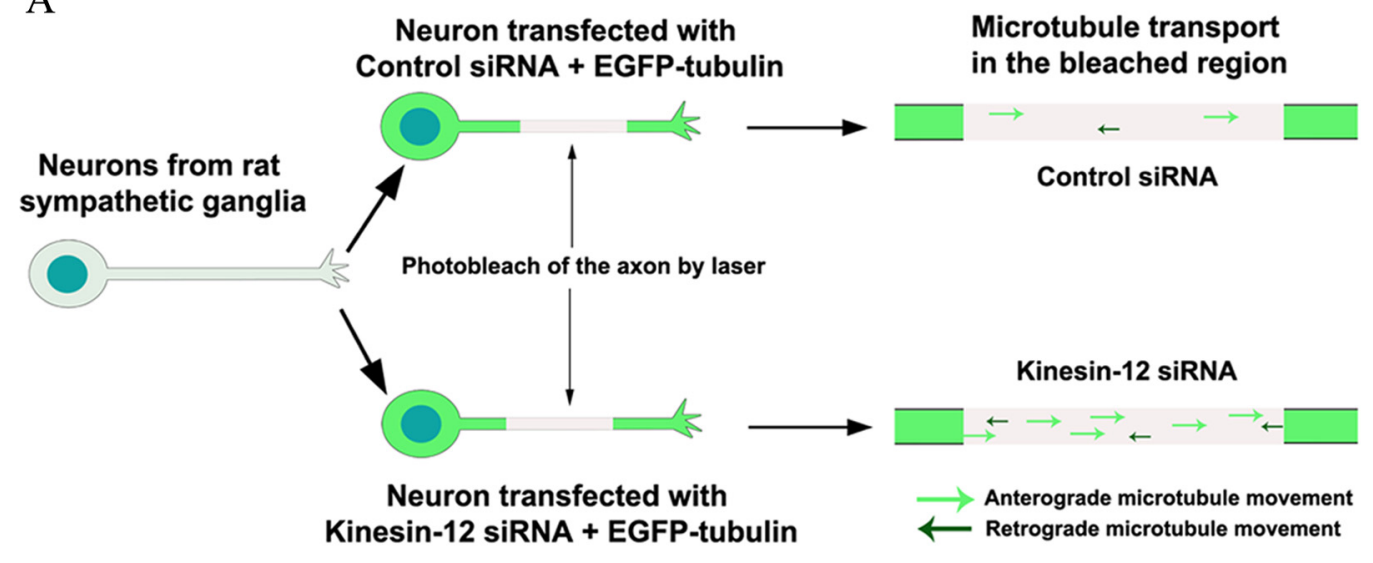

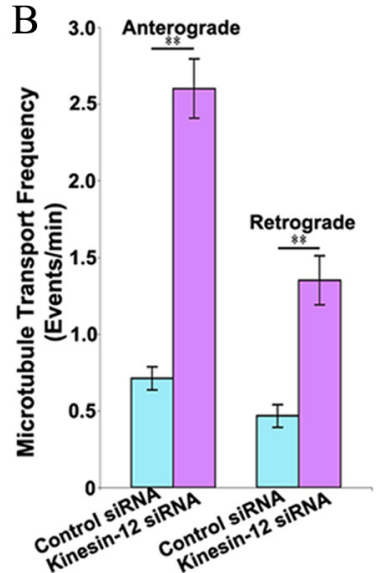

Figure 5. Depletion of kinesin- 12 from sympathetic neurons enhances the frequency of transport of short microtubules in both directions. A, Schematic illustration of the strategy for observing the transport of short microtubules within the axons of cultured neurons transfected with control siRNA and kinesin-12 siRNA. B, Quantification of transport frequency of short microtubules in axons of neurons transfected with kinesin-12 siRNA and control siRNA. Data are represented as mean \pm SE. The frequency of anterograde transport of short microtubules in axons per minute in control neurons is $0.7 \pm 0.1$ and in kinesin-12-depleted axons is $2.6 \pm 0.2$, and the frequency of retrograde transport in control neurons is $0.5 \pm 0.1$ and in kinesin-12-depleted neurons is $1.4 \pm 0.2 ; n=$ $10 ;{ }^{* *} p<0.01$.

were released from the substrate and replated at lower density on glass-bottomed $35 \mathrm{~mm}$ culture dishes and allowed to grow axons anew for $24 \mathrm{~h}$. With regard to PNS sympathetic neuronal cultures, the morphology of the neurons depleted of kinesin-12 was markedly different from controls, with the kinesin-12-depleted neurons displaying notably longer axons (Fig. $2 B-D$ ). The axons of kinesin-12-depleted neurons were also less branched and displayed fewer filopodia (Fig. 2E). Quantification of the morphological changes shown in Figure 2, D and E, revealed an almost 95.3\% increase in axonal length (see figure legend for statistics), and the axonal branches and filopodia were $66.3 \%$ and $51.3 \%$ diminished, relative to the control, respectively (see figure legend for statistics). The greater length of the axons is reminiscent of results obtained with inhibition or depletion of kinesin-5 (Haque et al., 2004; Yoon et al., 2005; Myers and Baas, 2007), but the effect on branching is the opposite.

\section{Depletion of kinesin-12 also impacts CNS neuronal morphology}

In our earlier work, we reported evidence suggesting that kinesin-12 may be able to interact with actin filaments (Buster et al., 2003). For this reason, we also used CNS cortical neurons, mainly because they undergo additional actin-dependent morphological behaviors not observed with the sympathetic neurons in culture. Specifically, they generate collateral branches along their length, a process that requires the interplay between microtubules and actin filaments (Dent and Kalil, 2001), and they generate growth-cone-like waves that move anterogradely down the axon's length (Ruthel and Banker, 1999; Flynn et al., 2009; Tint et al., 2009). [The branches from the axons of sympathetic neuron result almost entirely from growth cone bifurcation, and no such waves are seen moving along their axons.] The cortical neurons were transfected with the kinesin-12 siRNA for $3 \mathrm{~d}$, after which the level of kinesin-12 was decreased by almost 90\% (Fig. 3A). In cortical neurons depleted of kinesin-12, we observed a $21.2 \%$ increase in axonal length relative to controls (see figure legend for statistics), which is notable but less dramatic than the effect with the sympathetic neurons (Fig. $3 B, C$ ). With regard to collateral branches, filopodia along the axon's length, and the growthcone-like waves, there were diminutions compared to controls of $48.5 \%, 43.3 \%$, and 65.8\%, respectively (Fig. 3B, C). Quantifica- tion of the morphological changes is shown in Figure 3, D and $E$ [see figure legend for statistics and other details; growth-conelike waves were identified by their morphology and staining for cofilin; see Flynn et al. (2009)].

\section{Depletion of kinesin-12 inhibits growth cone turning}

To test whether kinesin-12 is involved in growth cone turning, we used the "border assay," which uses a glass coverslip with a sharp border between a laminin-coated side and a poly-DL-lysinecoated side (Turney and Bridgman, 2005; Nadar et al., 2008). When neurons are plated on the laminin side, growth cones normally turn when they reach the border. Indeed, almost all axons turned at the border in the case of control neurons (Fig. 4A). However, very few axons of the kinesin-12-depleted neurons turned (Fig. $4 B$ ). Instead, the growth cones ignored the border, crossed it, and continued growing on the polylysine side. This effect was quantified by measuring the fluorescence for the immunostained axonal microtubules on each side of the border (Fig. 4C). The ratio of microtubule fluorescence per unit pixel area of axons that crossed the border and axons that turned at the border showed $480 \%$ increase in kinesin-12-depleted neurons as compared to the control neurons (see figure legend for statistics). Notably, this effect is essentially the same as observed in our earlier study on neurons in which we depleted or inhibited kinesin-5 in these neurons (Nadar et al., 2008).

\section{Depletion of kinesin-12 increases frequency of microtubule transport in the axon}

The earliest speculations on the function of kinesin-12 in the mitotic spindle were that kinesin-12 keeps the half-spindles separated by opposing the forces generated by other motors that would otherwise draw the half-spindles together (Wittmann et al., 1998; Rogers et al., 2000). On this basis, we speculated that kinesin-12 might similarly oppose microtubule movements by other motors in the axon (Buster et al., 2003). Since then, we found that inhibiting kinesin-5 actually elicited the effects on the microtubule array that we might have predicted of inhibiting kinesin-12 (Myers and Baas, 2007; Nadar et al., 2008). Within the axon, only very short microtubules undergo rapid concerted transport, with about two-thirds of the movements in the anterograde direction and one-third in the retrograde direction (Wang 
and Brown, 2002; Hasaka et al., 2004; He et al., 2005). When kinesin-5 was inhibited or depleted, the frequency of these transport events increased (in both directions) (Myers and Baas, 2007). There was no change in the rate of transport of individual microtubules.

Here, we used the same live-cell imaging paradigm to study microtubule transport in the axons of kinesin-12-depleted rat sympathetic neurons. The method involves expression in the neurons of EGFP-tubulin, followed by photobleaching a $40 \mu \mathrm{m}$ region along the axon's length. Then, fluorescent microtubules from flanking unbleached regions are observed to move through the bleached zone (Fig. 5A). As with the kinesin-5 results, there was no change in the rate of the movements, but there were marked elevations in the frequency of movements in both directions. With kinesin-12 depletion, there were $266 \%$ and $187 \%$ increases in the anterograde and retrograde directions, respectively (Fig. 5B; see legend for statistics). This result is similar but not identical to the result with kinesin-5 inhibition, in which we observed an $\sim 79.5 \%$ increase in the anterograde direction and a $72.1 \%$ increase in the retrograde direction (Myers and Baas, 2007). Thus, it would appear that both motors are important for limiting the number of short microtubules moving within the axon.

\section{Kinesin-12 and kinesin-5 function independently of one another}

Although we have used kinesin-5 siRNA in past studies, a great deal of our work on this motor has used the allosteric kinesin-5 inhibitor called monastrol. Notably, kinesin- 5 depletion by siRNA results in essentially the same impact as monastrol treatment on microtubule transport and distribution, the same phenotypic effects on axonal length and morphology, and the same impact on growth cone turning (Haque et al., 2004; Myers and Baas, 2007; Nadar et al., 2008). Given that kinesin-12 does not share the unique large loop L5 region of kinesin- 5 targeted by the drug, we would not have predicted these drugs to inhibit kinesin12. However, the remarkable similarities between the phenotypes and especially the effects on microtubules in monastrol-treated cultures and kinesin-12-depleted cultures led us to wonder whether monastrol might inhibit kinesin-12 as well as kinesin-5. Curiously, although monastrol has been tested and found not to inhibit a wide array of other kinesins (DeBonis et al., 2003; Tcherniuk et al., 2010), it has never been tested on kinesin-12. So, here, we used the same approach as has been used in the past to test whether known kinesin-5 inhibitors also inhibit kinesin-12 (Mayer et al., 1999). This was done by measuring the inhibition of the basal and microtubule-stimulated ATPase activities of kinesin-12 in the presence of increasing concentrations of monastrol (and also STLC, another anti-kinesin-5 drug that is currently more often used than monastrol by many investigators because it is potent in the low nanomolar range). Kinesin-5 was used as a positive control. Whereas monastrol inhibits kinesin-5 activities with $\mathrm{IC}_{50}$ values of $7.7 \pm 1.1 \mu \mathrm{M}$ and $31.2 \pm 4.4 \mu \mathrm{M}$ for the basal and microtubule-stimulated ATPase activities, respectively, kinesin-12 was not inhibited at concentrations up to 100 $\mu \mathrm{M}$, indicating the specificity of monastrol for kinesin-5 (Fig. $6 A, B)$. This result confirmed that kinesin-12 is not inhibited by monastrol, even when used at the high concentrations (e.g., 100 $\mu \mathrm{M}$ ) in our cell biological studies. STLC was also shown in our study not to inhibit kinesin-12 (Fig. $6 C, D$ ).

Interestingly, as we were conducting these studies on neurons, two articles were published indicating overlapping phenotypes of kinesin-12 and kinesin-5 inhibition during mitosis (Tanenbaum
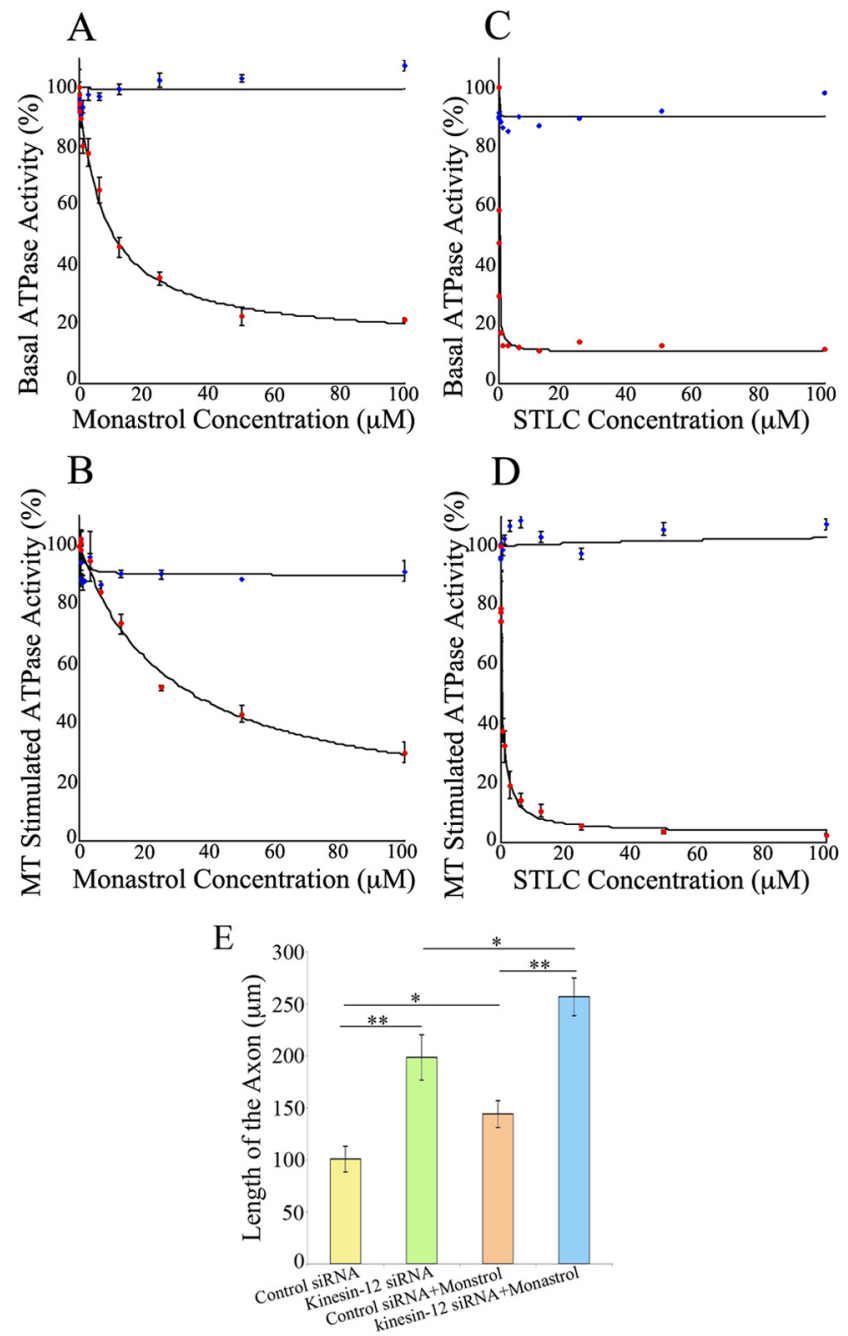

Figure 6. Kinesin-12 and kinesin-5 function independently of one another in affecting axonal length. $\boldsymbol{A}$, Plot for the inhibition of the basal ATPase activity of kinesin-5 (red) and kinesin-12 (blue) in the presence of increasing monastrol concentrations. Kinesin-5 is inhibited by monastrol with an $\mathrm{IC}_{50}$ value of $7.7 \pm 1.0 \mu \mathrm{m}$, while kinesin- 12 is not affected up to a concentration of $100 \mu \mathrm{m} . \boldsymbol{B}$, Inhibition of the microtubule-stimulated ATPase activity of kinesin-5 (red) and kinesin-12 (blue) by monastrol. $\boldsymbol{C}$, Concentration-response plot for the inhibition of the basal ATPase activity of kinesin-5 (red) and kinesin-12 (blue) by STLC. STLC does not affect kinesin- 12 but strongly inhibits kinesin-5 with an $\mathrm{IC}_{50}$ of $114 \pm 9.8 \mathrm{~nm}$. $\boldsymbol{D}$, Inhibition of kinesin-5 (red) and kinesin-12 (blue) by STLC in the presence of microtubules. $\boldsymbol{E}$, Quantification of axonal length in rat sympathetic ganglia shows significantly longer axons in neurons depleted of kinesin- 12 and treated with monastrol compared to either neurons transfected with control siRNA or treated with monastrol. Data are represented as mean $\pm \mathrm{SE}$; control siRNA $=100.7 \pm 12.4 \mu \mathrm{m}$; kinesin-12 siRNA $=198.4 \pm 20.6 \mu \mathrm{m}$; control siRNA + monastrol $=143.9 \pm 13.0 \mu \mathrm{m}$; kinesin- $12 \mathrm{siRNA}+$ monastrol $=256.6 \pm 17.6 \mu \mathrm{m} ; n=17$. ${ }^{*} p<0.05,{ }^{* *} p<0.01$.

et al., 2009; Vanneste et al., 2009). It was reported that cells depleted of kinesin- 5 can recover from their monastrol phenotype if kinesin-12 is overexpressed (Tanenbaum et al., 2009). Thus, apparently the functions of these two motors are similar enough that, at least in some circumstances, one can substitute for the other. We wondered whether the two motors in neurons might produce such similar phenotypes, when inhibited together, because they somehow depend on each other. However, arguing against this, when we combined monastrol treatment with kinesin-12 inhibition, we found that axons grew faster than with either treatment alone. As shown in Figure $6 E$, the elevations above control levels were roughly additive, which suggests that 

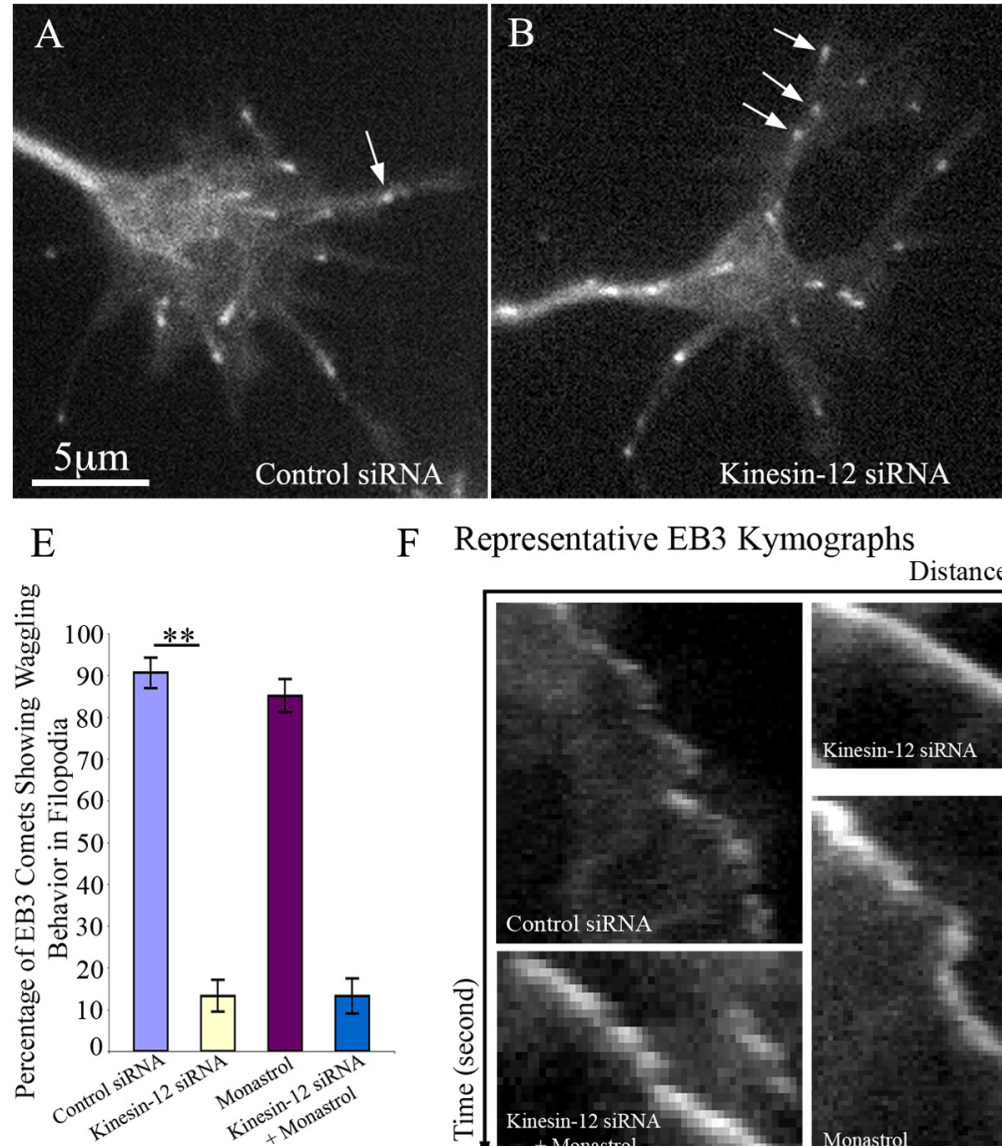

F Representative EB3 Kymographs

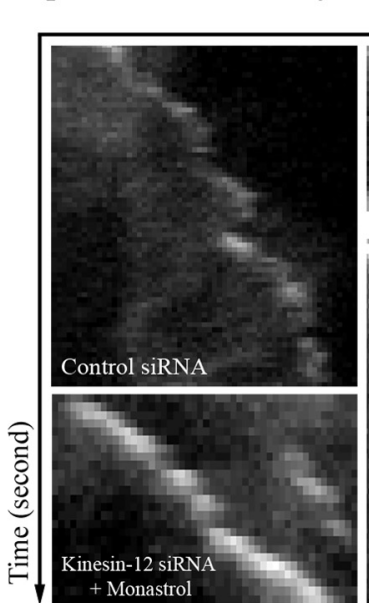

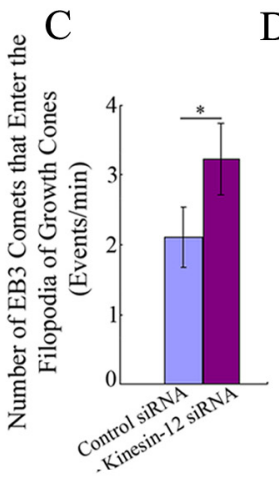

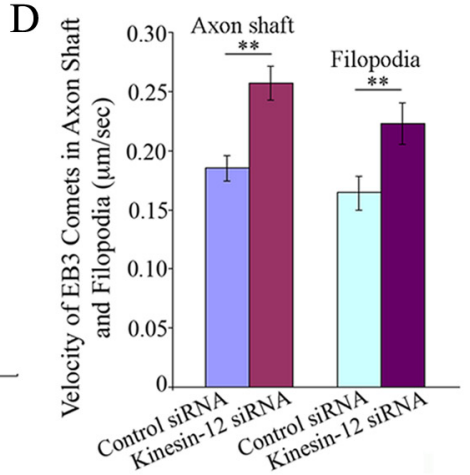

G

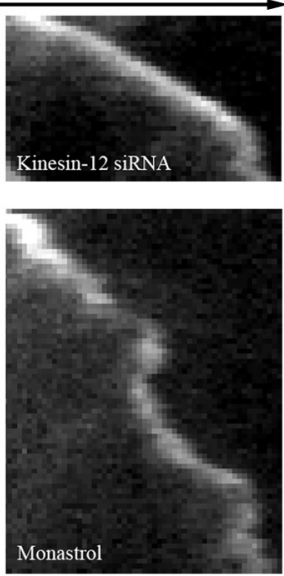

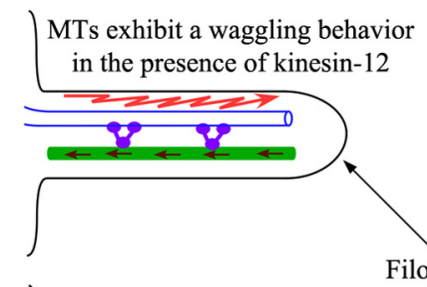

lopodia

MTs advance smoothly in the absence of kinesin-12

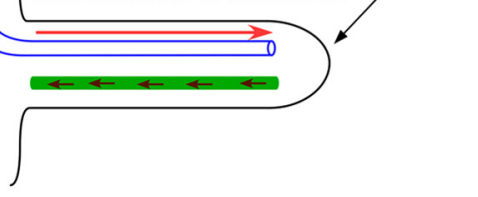

F-actin [ु MT Kinesin-12 $\rightarrow$ Direction of $\rightarrow$ Actomycin

Figure 7. Effects of kinesin-12 depletion on the behavior of microtubules in growth cones, as revealed by EGFP-EB3 comets. $A, B$, Example micrographs of EGFP-EB3 comets in a growth cone of a control neuron $(\boldsymbol{A})$ and a neuron depleted of kinesin-12 by siRNA $(\boldsymbol{B})$, where the white arrows indicate EB3 comets in a control and experimental filopodium. Note that in the control filopodium, there is one comet, whereas in the filopodium from the kinesin-12-depleted neuron, there are three. $C$, Quantification of the number of EB3 comets that enter the filopodia in growth cones per minute. Data are represented as mean $\pm \mathrm{SE}$; control siRNA $=2.11 \pm 0.44, n=31$; kinesin- 12 siRNA $=3.23 \pm 0.51, n=34 ;{ }^{*} p<0.05$. D, Quantification of the velocity of EB3 comets in the axonal shaft and in the filopodia. Velocity of EB3 comets in the axonal shaft is control siRNA $=0.2 \pm 0.01 \mu \mathrm{m} / \mathrm{s}$; kinesin-12 siRNA $=0.3 \pm 0.01 \mu \mathrm{m} / \mathrm{s}$; velocity of EB3 comets that enter the filopodia in control siRNA $=0.2 \pm 0.01 \mu \mathrm{m} / \mathrm{s}, n=31$; kinesin-12 siRNA $=0.2 \pm 0.02 \mu \mathrm{m} / \mathrm{s}, n=30 ;{ }^{*} p<0.05$, ${ }^{* *} p<0.01$. E, Graph showing percentage of EB3 comets exhibiting waggling behavior in filopodia. The data indicate that the percentage of comets exhibiting waggling is significantly less in filopodia of kinesin-12-depleted neurons as compared to filopodia of control neurons. Data are represented as mean $\pm \mathrm{SE}$; control siRNA/DMSO $=90.7 \pm 3.7$; kinesin- 12 siRNA/DMSO $=13.3 \pm 3.8 ;$ control siRNA/monastrol $=85.1+4.0 ;$ kinesin- 12 siRNA $/$ monastrol $=13.3 \pm 4.2 . n=$ $10,{ }^{* *} p \leq 0.001$.F, Kymographs of EGFP-EB3 comets in the filopodia of neurons transfected and treated with control siRNA/DMSO, kinesin-12 siRNA/DMSO, kinesin-12 siRNA/monastrol, and control siRNA/monastrol. The EB3 comets in filopodia of control siRNA-transfected neurons show a waggling behavior as revealed here by the jagged kymograph, whereas the EB3 comets in kinesin-12 siRNA-transfected filopodia do not show waggling, as revealed here by the smooth kymograph. The comets in kinesin-12 siRNA and monastrol-treated filopodia show behavior similar to kinesin- 12 siRNA-transfected filopodia, whereas the comets in control siRNA and monastrol-treated filopodia shows behaviors similar to the comets in control siRNA and DMSO-treated filopodia. G, Schematic of our proposal for the behavior of microtubules in the filopodia. Microtubules (shown in blue) in the filopodium assemble along the actin bundle (green) and exhibit waggling behavior (red trace) due to the retrograde actomyosin forces (brown arrows) that act in the direction opposite to that of microtubule assembly. We propose that kinesin-12 integrates the microtubule with the actin bundle in the filopodium, thereby resulting in the waggling behavior that is lost when kinesin-12 is absent.

each motor has its own independent effects to limit the growth of the axon.

\section{Depletion of kinesin-12 impacts microtubule behaviors in the growth cone as well as the size of the growth cone}

The reason why kinesin-5 inhibition causes growth cones to fail to turn is readily observed if the neurons are transfected to express EGFP-EB3. EB3 is a microtubule end-binding protein that associates with the plus-end of the microtubule during bouts of assembly, and hence the GFP protein reveals microtubule behaviors in live-cell imaging in the form of fluorescent "comets" at the plus ends of the assembling microtubules (Stepanova et al., 2003). When kinesin-5 is inhibited, there is a notable increase in the numbers of comets entering the peripheral domain of the growth cone and an increase in the rate of the comets as well (Nadar et al., 2008). As shown in Figure 7A-C, the growth cones of kinesin-12-depleted neurons show qualitatively the same effect. Quantitative analysis of the number of EB3 comets that enter filopodia of growth cones per minute showed an increase of 52\% in the case of neurons depleted of kinesin-12 compared to control (Fig. 7C, see figure legend for details). Depletion of kinesin-12 also resulted in a $39 \%$ increase in the velocity of EB3 comets in the axonal shaft and a $36 \%$ increase in the velocity in the filopodia (Fig. 7D, see figure legend for statistics).

More detailed analyses on the EGFP-EB3 comets revealed a difference in microtubule behaviors in growth cones of neurons depleted of kinesin-12 versus neurons treated with monastrol. In control neurons, the microtubules show a subtle but consistent "waggling" behavior in filopodia, which presumably reflects the retrograde flow of the actin bundle along which the microtubule assembles. This effect was obliterated with the depletion of kinesin-12, but not with the inhibition of kinesin-5 (shown in 

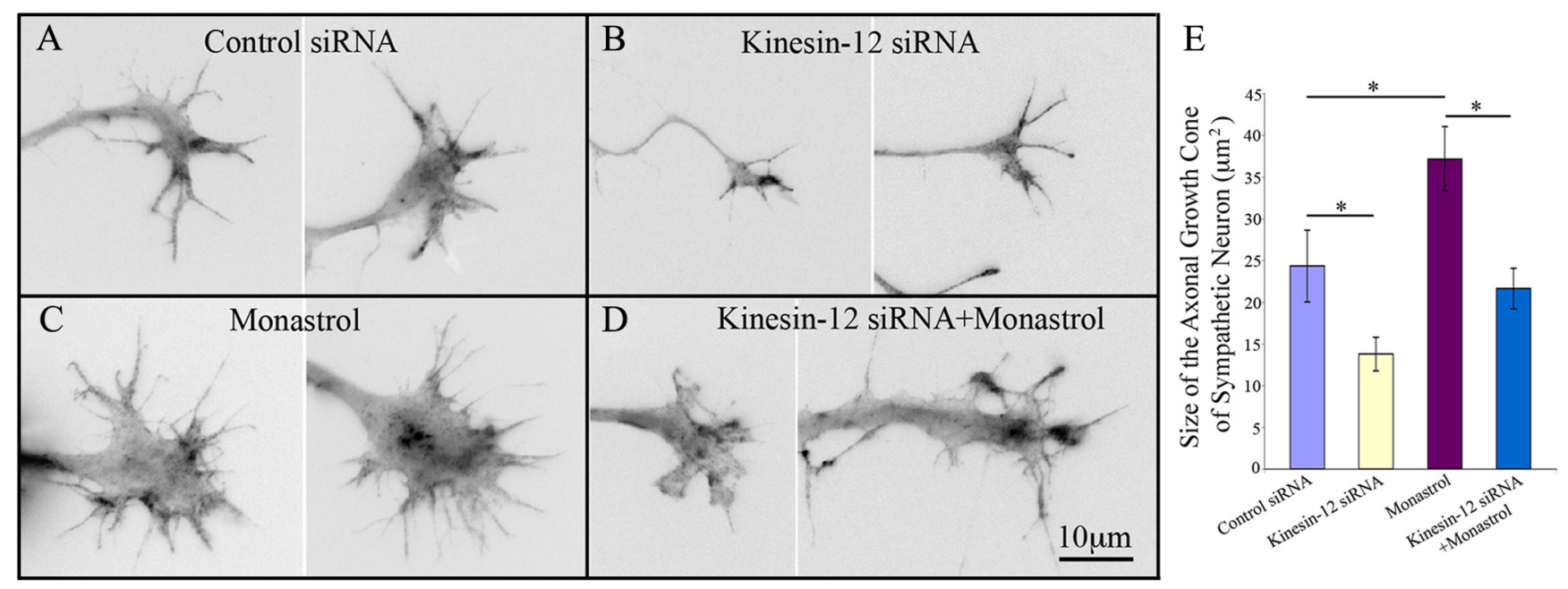

Figure 8. Depletion of kinesin-12 has an effect on growth cone size opposite to that of inhibition of kinesin-5. $A-D$, Representative fluorescence images of growth cones stained with Alexa Fluor 488-Phalloidin to reveal actin filaments. Images were inverted to show the morphologies more clearly. $\boldsymbol{A}$, Control siRNA and DMSO; $\boldsymbol{B}$, kinesin-12 siRNA and DMSO; $\boldsymbol{C}$, control siRNA and monastrol; D, kinesin-12 siRNA and monastrol. Scale bar, $10 \mu \mathrm{m}$. $\boldsymbol{E}$, Quantification of growth cone size of cultured sympathetic neurons revealed that depletion of kinesin-12 and inhibition of kinesin-5 had

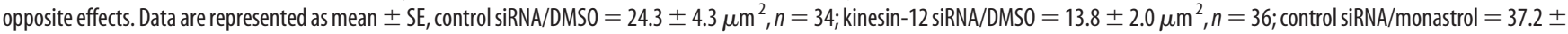
$3.9 \mu \mathrm{m}^{2}, n=45 ;$ kinesin-12 siRNA/monastrol $=21.6 \pm 2.4 \mu \mathrm{m}^{2}, n=34 ;{ }^{*} p<0.05$.

Fig. 7 E, F; and displayed schematically in Fig. 7G). The percentage of EB3 comets showing the waggling behavior in kinesin-12 siRNA/DMSO-treated neurons was significantly lower than in control siRNA/DMSO-treated neurons $(p<0.001)$. There was no significant difference between the monastrol-treated neurons and control siRNA/DMSO-treated neurons $(p>0.05)$ or between kinesin-12 siRNA/DMSO-treated neurons and kinesin-12 siRNA/monastrol-treated neurons $(p>0.05)$ in terms of the percentage of EB3 comets showing the waggling behavior. There was a significant decrease of the percentage of EB3 comets showing the waggling behavior in the kinesin-12 siRNA/ monastrol-treated neurons as compared to the control siRNA/ DMSO-treated and control siRNA/monastrol-treated neurons $(p<0.001)$. Representative movies of the different treatments are available at www.jneurosci.org as supplemental material (Movies 1-4; see movie legends for details).

With regard to growth cone size, we observed opposite effects from depleting kinesin-12 and inhibiting kinesin-5. Compared to the control, depletion of kinesin-12 showed $43.2 \%$ decrease of mean size of growth cone, while inhibition of kinesin- 5 produced a $53.1 \%$ increase, as shown in Figure $8 \mathrm{~A}-E$. There was no significant difference compared to control when neurons were treated with kinesin-12 siRNA combined with monastrol [although the morphology is somewhat different; with both motors suppressed, the growth cone looks more flattened (Fig. 8D)]. These effects may be related to kinesin-5's microtubule cross-linking properties relative to kinesin-12, given that more tightly bundled microtubules would presumably lead to more compact growth cones, and to kinesin-12's potential ability to interact with actin filaments (see next section), a major determinant of growth cone shape.

\section{Kinesin-12 coimmunoprecipitates with actin, but kinesin-5 does not}

The observed differences in the phenotypes of neurons in which we depleted kinesin-12 or inhibited kinesin- 5 are consistent with a scenario whereby the unique effects of kinesin-12 result from its ability to interact with actin. Our evidence for this was the fact that in RFL-6 rat fibroblasts, the staining with an antibody for kinesin-12 colocalized mainly with actin bundles in interphase as well as with the cleavage furrow in telophase (Buster et al., 2003). We noted that kinesin-12 has a myosin-like sequence within its stalk region, and posited that it is perhaps through this domain that kinesin-12 elicits such an interaction. However, other possibilities exist for the actin-like immunostain pattern of kinesin-12 in these fibroblasts. The antibody that we found most effective for immunostaining was generated against the stalk domain of kinesin-12, and produced an extra band of unknown identity on Western blots (Buster et al., 2003). Given that the molecular weight of the extra band is consistent with myosins, we wondered whether the actin-like staining pattern at some phases of the cell cycle might relate to cross-reactivity of the antibody with myosins rather than a bona fide colocalization of kinesin-12 with actin. We have now sequenced this extra band and found that it indeed corresponds to MYH9 and MYH10 (both of which are myosin-II family members). To ascertain whether any of the actin-like staining is actually due to kinesin-12, we depleted kinesin-12 from the fibroblasts using siRNA and then immunostained the cells with the same antibody. We found that the actin-like staining was either completely eliminated from cells or markedly reduced. Any remaining filamentous staining was typically observed in the more peripheral regions of the cells. For quantification, we scored cells as positive if they had any detectable filamentous quality to their staining, or negative if the staining was entirely diffuse in nature. Thus, by these criteria (which err on the side of caution, given that we scored cells as positive even if the filamentous staining was down substantially but not completely eliminated), the loss was $\sim 57 \%$ ( $p<0.0001 ; \chi^{2}$ test) (Fig. $9 A-C)$. These results indicate that while some of the actin colocalization is not due to kinesin-12, much of it is. Consistent with this, previous studies on kinesin-12 immunostaining in other species (Rogers et al., 2000) using different antibodies also reported the localization of kinesin-12 to the telophase cleavage furrow.

We next wished to further test for a potential interaction between kinesin-12 and actin, using a biochemical approach. For this, we used our new rKinesin-12 antibody, which is raised against the tail domain and hence does not recognize myosins. 
This antibody is not optimal for immunostaining, but is quite specific to kinesin12 , as it produces only one band on Western blots. We found that kinesin-12 coimmunoprecipitates with actin in a widely used and reliable coimmunoprecipitation assay (Fig. 9D), but that kinesin-5 does not (Fig. 9E). This result confirms that kinesin-12, at least under certain circumstances, can interact with actin, although the question remains as to whether this interaction is direct or indirect.

\section{Discussion}

Our original goal in studying mitotic kinesins in neurons was to identify the motors that transport microtubules in axons and dendrites (Baas, 1999). With regard to the axon, our hypothesis has been that cytoplasmic dynein and a small number of mitotic kinesins share this responsibility (Baas et al., 2006). Given that depletion of cytoplasmic dynein reduces the anterograde movements by half with no apparent reduction in the retrograde movements (He et al., 2005), we concluded that there is a need for kinesins to participate in the transport of microtubules in both directions. We have now studied kinesin-5 and kinesin-12. Interestingly, depletion or inhibition of these motors has no detrimental effect on the frequency of short microtubule transport (Myers and Baas, 2007), but instead, produces a marked increase in transport frequency. Thus, our hypothesis regarding these motors and their role in the transport of axonal microtubules needs to be revised. One possibility is that cytoplasmic dynein is actually responsible for a greater share of the burden than our studies thus far have suggested, given that we have generally depleted only $\sim 85 \%$ of the dynein in these experiments. Another possibility is that some of the nonmitotic kinesins may be able to participate in the transport of microtubules. Consistent with this, recent studies indicate that kinesin- 1 is able to transport microtubules in other cell types (Jolly et al., 2010).

Since our original finding that inhibition of kinesin-5 enhances bidirectional microtubule transport in the axon (Myers and Baas, 2007), the underlying mechanistic explanation for this has been elusive. Kinesin- 5 is a homotetramer with four motor domains projected outward (Kashina et al., 1996), thus enabling it to move along two neighboring microtubules toward their plus ends (Kapitein et al., 2008). If the microtubules are oppositely oriented, the result would be to move them apart. If the microtubules have the same orientation, the homotetramer would presumably move along both of them, perhaps helping to align them, but not immobilizing them. In the case of the bipolar kinesin-12, less is known about how it generates forces between microtubules, but it apparently works in conjunction with a microtubule-binding protein called TPX2 to mimic the manner by which kinesin-5 functions (Tanenbaum et al., 2009). This is feasible in neurons, given that TPX2 has been identified in the cytoplasm of neurons (Mori et al., 2009). Both motors can presumably cross-link microtubules, but to cross-link them does not mean to immobilize them, unless the motor is in a rigor state, and there is no real precedent for this occurring in living cells, to the
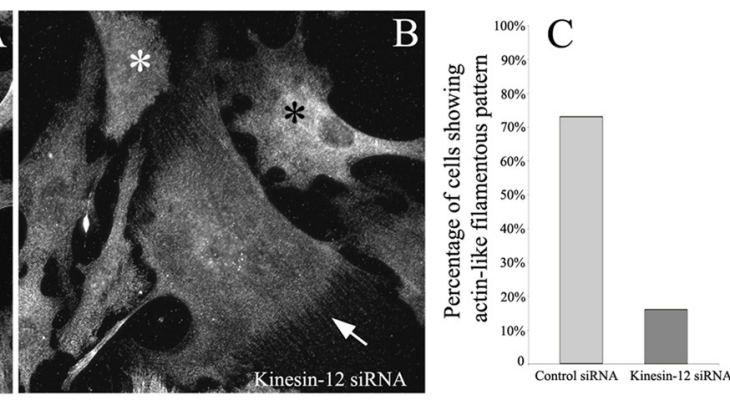

$\mathrm{E}$
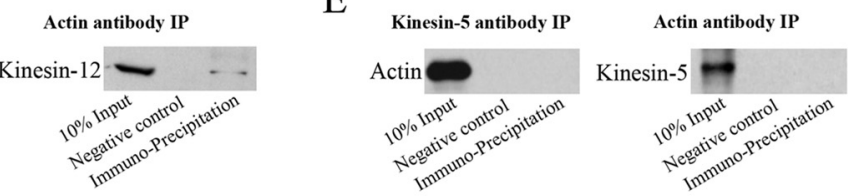

Figure 9. Evidence for an interaction of kinesin-12 but not kinesin-5 with actin. $\boldsymbol{A}-\boldsymbol{C}$, Experiment in which the kinesin-12 antibody we previously used for immunostaining RFL-6 fibroblasts (Buster et al., 2003) was used to immunostain control cultures A) as well as cultures that had been depleted of kinesin-12 by siRNA (B). Scale bar, $100 \mu \mathrm{m}$. The staining typically shows a strong siRNA: $16 \%, n=266 ; p<0.0001$ ( $\chi^{2}$ test). $D$, Western blot showing the positive results of the coimmunoprecipitation assay for kinesin-12 and actin. $\boldsymbol{E}$, Western blot showing the negative results of coimmunoprecipitation of kinesin-5.

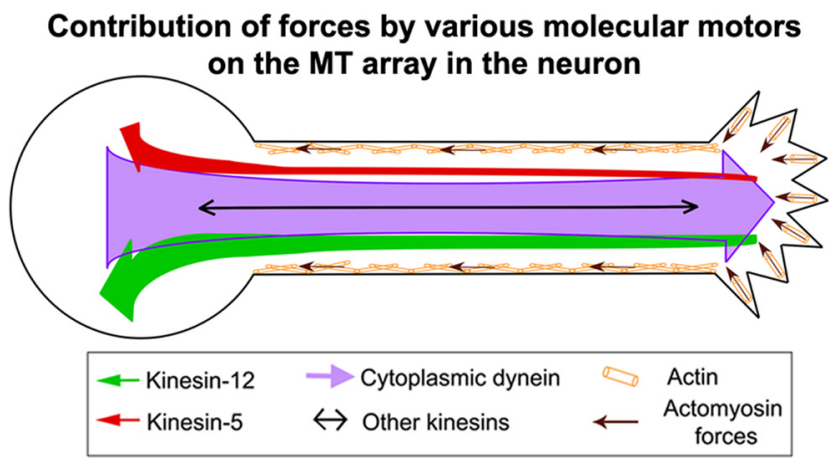

Figure 10. A model for how forces imposed by various molecular motors act on the microtubule array of the axon. We posit a model wherein cytoplasmic dynein imposes forces that pull on the microtubules in an anterograde direction, while kinesin- 5 and kinesin- 12 impose forces that pull in the retrograde direction. Actomyosin forces push back on the microtubules in the growth cone and also impose forces along the axonal shaft that compress the microtubules toward the cell body. A variety of other kinesins impose forces on the microtubules in both directions, depending on the orientation of the motor. Kinesin- 12 forces are stronger than kinesin- 5 forces during development (depicted here by the different thicknesses of the force arrows), but kinesin- 12 levels diminish more as development ensues than do kinesin- 5 levels. The kinesin- 12 and kinesin- 5 forces are stronger in the cell body than in the axon itself, thereby regulating the entry of short microtubules into the axons. The forces exerted by cytoplasmic dynein are more uniform throughout the cell body and axons.

best of our knowledge. Now that we know that kinesin-12 imposes an even more potent effect to diminish short microtubule transport in the developing axon than kinesin-5, the question becomes more urgent as to how exactly these two very different motors are doing this.

A potential clue comes from recent studies in which we quantified microtubule transport in the axon after overexpressing spastin, a potent microtubule-severing protein (Qiang et al., 2010). This regime resulted in higher numbers of short microtubules throughout the neuron. The frequency of short microtu- 
bule transport in the axon was notably increased in both directions and about equally in both directions. These results suggest that it may not be motors acting as brakes in the axon that controls the number of short microtubules in transit, but rather it may be the number of short microtubules in the axon that determines the frequency of observable transport events. Thus under normal conditions, it may be that kinesin-5 and kinesin-12 are imposing a limit on the number of short microtubules that can transit into the axon from the cell body, rather than serving as brakes within the axon itself. Mechanistically, this might make more sense because microtubules are situated in all manner of configurations in the cell body (Yu et al., 2001), so there are a number of different possibilities for how forces generated by kinesin-5 and kinesin-12 could work in opposition to cytoplasmic dynein to limit the number of microtubules than can move into the axon.

A marked increase in the vitality of short microtubule transport could be a significant factor in why suppressing either kinesin- 5 or kinesin- 12 causes axons to grow faster. However, the profound phenotypes we observed when these motors are depleted or inhibited may be more attributable to their effects on the longer stationary microtubules in the axon. Assuming kinesin-5 and kinesin-12 can be transiently immobilized against other structures, long microtubules interacting with their motor domains would be pulled toward the cell body. Taking into account our previous findings on other motors, cytoplasmic dynein would simultaneously pull on the microtubules in the opposite direction (Myers et al., 2006). In order for the axon not to retract, the sum of these forces (and perhaps forces generated by other kinesins) must be greater than the contractile forces generated by the actomyosin system along the axonal shaft. If this is the case, the axon grows. If it is not the case, the axon retracts. Removing either kinesin-5 or kinesin-12 shifts the balance toward growth, which is why the axon becomes longer when either of these motors is depleted or inhibited. This scenario (shown schematically in Fig. 10) can also explain the effects on the growth cone, wherein the actomyosin forces are pushing the microtubules backward toward the axonal shaft, while dynein-driven forces are pulling the microtubules forward from the axonal shaft into the peripheral domain of the growth cone. Kinesin-5 and kinesin-12 both oppose the forces of cytoplasmic dynein to enable microtubules to enter the peripheral domain and invade filopodia. The forces would have to be spatially regulated (Nadar et al., 2008), to direct microtubules into certain filopodia but not others during normal growth cone turning. If either kinesin- 5 or kinesin- 12 is inhibited, microtubules enter the filopodia without proper spatial control, and the growth cone fails to turn properly.

Overlapping functions for kinesin-5 and kinesin-12 in the neuron are consistent with recent reports of kinesin- 12 being able to compensate for loss of kinesin-5 during mitosis (Tanenbaum et al., 2009). However, the phenotypes observed when one or the other motor is suppressed in neurons are not identical in every way. For example, suppression of kinesin-5 increases axonal branching, while suppression of kinesin-12 decreases axonal branching. Considering only microtubule-microtubule interactions, kinesin-12 should suppress axonal branching, as does kinesin-5, by pulling the microtubules backward. The unique feature of kinesin-12 that might account for the reverse phenotype is the potential for kinesin-12 to interact with the actin cytoskeleton. We previously reported that kinesin-12 has a myosin-like domain in its stalk region (Buster et al., 2003), and this might explain its positive impact on axonal branching, given that axonal branch formation requires interactions between mi- crotubules and filamentous actin (Dent and Kalil, 2001). In support of this idea, at least one antibody to kinesin-12 produces staining that colocalizes with actin filaments at certain stages of the cell cycle in fibroblasts, although this result needs to be interpreted with caution because the antibody also recognizes myosin-II family members (but also see experiments in Fig. 9A$C)$. Additional support for an interaction with actin comes from our finding that kinesin-12 coimmunoprecipitates with actin, and depletion of kinesin-12 from neurons results in smaller growth cones and fewer growth-cone-like waves along the axon. Finally, detailed analyses of microtubules assembling along the actin bundle in filopodia suggest that the microtubule is normally linked to the actin bundle (as demonstrated by a subtle "waggling" effect on the microtubule's trajectory) but loses this linkage in the absence of kinesin-12 (Fig. 7F). None of these observations are true of kinesin-5.

To the best of our knowledge, there are just a few examples in the literature of kinesins interacting with actin. A plant-specific member of the kinesin-14 family in cotton has been shown to strongly interact with actin (Xu et al., 2009), and the CHO1 variant of the MKLP1 (kinesin-6) family has a domain that interacts with actin (Kuriyama et al., 2002). Other kinesins may interact with actin indirectly, through common binding partners (Medina et al., 2008). Given that kinesin-12 has a myosin-like domain, its interaction with actin could be direct, but it is worth noting that we have never observed an immunostain pattern in neurons with any kinesin-12 antibody that suggests an actin-like distribution. Thus, whatever the interaction may be, direct or indirect, we would surmise that it is tightly regulated and not "always on." The fact that kinesin-12 is expressed early in neuronal development but not later is consistent with a role for kinesin-12 in actin-based structures and events that are especially predominant during development, such as branch formation, growth cone form and function, and the propagation of growthcone-like waves within some axons.

In conclusion, we have reported new information on a little studied but functionally powerful molecular motor in developing axons. In addition to providing insights into kinesin- 12 itself, the results of these studies challenge some of our own previous views on how molecular motors impact both short and long microtubules in the axon.

\section{References}

Ahmad FJ, Hughey J, Wittmann T, Hyman A, Greaser M, Baas PW (2000) Motor proteins regulate force interactions between microtubules and $\mathrm{mi}-$ crofilaments in the axon. Nat Cell Biol 2:276-280.

Ahmad FJ, He Y, Myers KA, Hasaka TP, Francis F, Black MM, Baas PW (2006) Effects of dynactin disruption and dynein depletion on axonal microtubules. Traffic 7:524-537.

Baas PW (1999) Microtubules and neuronal polarity: lessons from mitosis. Neuron 22:23-31.

Baas PW, Vidya Nadar C, Myers KA (2006) Axonal transport of microtubules: the long and short of it. Traffic 7:490-498.

Buster DW, Baird DH, Yu W, Solowska JM, Chauvière M, Mazurek A, Kress M, Baas PW (2003) Expression of the mitotic kinesin Kif15 in postmitotic neurons: implications for neuronal migration and development. J Neurocytol 32:79-96.

DeBonis S, Simorre JP, Crevel I, Lebeau L, Skoufias DA, Blangy A, Ebel C, Gans P, Cross R, Hackney DD, Wade RH, Kozielski F (2003) Interaction of the mitotic inhibitor monastrol with human kinesin Eg5. Biochemistry 42:338-349.

DeBonis S, Skoufias DA, Indorato RL, Liger F, Marquet B, Laggner C, Joseph B, Kozielski F (2008) Structure-activity relationship of S-trityl-Lcysteine analogues as inhibitors of the human mitotic kinesin Eg5. J Med Chem 51:1115-1125. 
Dent EW, Kalil K (2001) Axon branching requires interactions between dynamic microtubules and actin filaments. J Neurosci 21:9757-9769.

Ferenz NP, Gable A, Wadsworth P (2010) Mitotic functions of kinesin-5. Semin Cell Dev Biol 21:255-259.

Flynn KC, Pak CW, Shaw AE, Bradke F, Bamburg JR (2009) Growth conelike waves transport actin and promote axonogenesis and neurite branching. Dev Neurobiol 69:761-779.

Haque SA, Hasaka TP, Brooks AD, Lobanov PV, Baas PW (2004) Monastrol, a prototype anti-cancer drug that inhibits a mitotic kinesin, induces rapid bursts of axonal outgrowth from cultured postmitotic neurons. Cell Motil Cytoskeleton 58:10-16.

Hasaka TP, Myers KA, Baas PW (2004) Role of actin filaments in the axonal transport of microtubules. J Neurosci 24:11291-11301.

He Y, Francis F, Myers KA, Yu W, Black MM, Baas PW (2005) Role of cytoplasmic dynein in the axonal transport of microtubules and neurofilaments. J Cell Biol 168:697-703.

Jolly AL, Kim H, Srinivasan D, Lakonishok M, Larson AG, Gelfand VI (2010) Kinesin-1 heavy chain mediates microtubule sliding to drive changes in cell shape. Proc Natl Acad Sci U S A 107:12151-12156.

Kaan HY, Ulaganathan V, Hackney DD, Kozielski F (2009) An allosteric transition trapped in an intermediate state of a new kinesin-inhibitor complex. Biochem J 25:55-60.

Kage K, Fujita T, Sugimoto Y (2005) Role of Cys-603 in dimer/oligomer formation of the breast cancer resistance protein BCRP/ABCG2. Cancer Sci 96:866-872.

Kapitein LC, Kwok BH, Weinger JS, Schmidt CF, Kapoor TM, Peterman EJ (2008) Microtubule cross-linking triggers the directional motility of kinesin-5. J Cell Biol 182:421-428.

Kashina AS, Baskin RJ, Cole DG, Wedaman KP, Saxton WM, Scholey JM (1996) A bipolar kinesin. Nature 379:270-272.

Kuriyama R, Gustus C, Terada Y, Uetake Y, Matuliene J (2002) CHO1, a mammalian kinesin-like protein, interacts with F-actin and is involved in the terminal phase of cytokinesis. J Cell Biol 156:783-790.

Lasek RJ (1986) Polymer sliding in axons. J Cell Sci Suppl 5:161-179.

Mayer TU, Kapoor TM, Haggarty SJ, King RW, Schreiber SL, Mitchison TJ (1999) Small molecule inhibitor of mitotic spindle bipolarity identified in a phenotype-based screen. Science 286:971-974.

Medina PM, Worthen RJ, Forsberg LJ, Brenman JE (2008) The actinbinding protein capulet genetically interacts with the microtubule motor kinesin to maintain neuronal dendrite homeostasis. PLoS One 3:e3054.

Mori D, Yamada M, Mimori-Kiyosue Y, Shirai Y, Suzuki A, Ohno S, Saya H, Wynshaw-Boris A, Hirotsune S (2009) An essential role of the aPKCAurora A-NDEL1 pathway in neurite elongation by modulation of microtubule dynamics. Nat Cell Biol 11:1057-1068.

Myers KA, Baas PW (2007) Kinesin-5 regulates the growth of the axon by acting as a brake on its microtubule array. J Cell Biol 178:1081-1091.

Myers KA, Tint I, Nadar CV, He Y, Black MM, Baas PW (2006) Antagonistic forces generated by cytoplasmic dynein and myosin-II during growth cone turning and axonal retraction. Traffic 7:1333-1351.
Nadar VC, Ketschek A, Myers KA, Gallo G, Baas PW (2008) Kinesin-5 is essential for growth-cone turning. Curr Biol 18:1972-1977.

Qiang L, Yu W, Liu M, Solowska JM, Baas PW (2010) Basic fibroblast growth factor elicits formation of interstitial axonal branches via enhanced severing of microtubules. Mol Biol Cell 21:334-344.

Rogers GC, Chui KK, Lee EW, Wedaman KP, Sharp DJ, Holland G, Morris RL, Scholey JM (2000) A kinesin-related protein, KRP(180), positions prometaphase spindle poles during early sea urchin embryonic cell division. J Cell Biol 150:499-512.

Ruthel G, Banker G (1999) Role of moving growth cone-like "wave" structures in the outgrowth of cultured hippocampal axons and dendrites. J Neurobiol 39:97-106.

Scholey JM (2009) Kinesin-5 in Drosophila embryo mitosis: sliding filament or spindle matrix mechanism? Cell Motil Cytoskeleton 66:500-508.

Stepanova T, Slemmer J, Hoogenraad CC, Lansbergen G, Dortland B, De Zeeuw CI, Grosveld F, van Cappellen G, Akhmanova A, Galjart N (2003) Visualization of microtubule growth in cultured neurons via the use of EB3-GFP (end-binding protein 3-green fluorescent protein). J Neurosci 23:2655-2664.

Tanenbaum ME, Macůrek L, Janssen A, Geers EF, Alvarez-Fernández M, Medema RH (2009) Kif15 cooperates with eg5 to promote bipolar spindle assembly. Curr Biol 19:1703-1711.

Tcherniuk S, van Lis R, Kozielski F, Skoufias DA (2010) Mutations in the human kinesin Eg5 that confer resistance to monastrol and S-trityl-1cysteine in tumor derived cell lines. Biochem Pharmacol 79:864-872.

Tint I, Jean D, Baas PW, Black MM (2009) Doublecortin associates with microtubules preferentially in regions of the axon displaying actin-rich protrusive structures. J Neurosci 29:10995-11010.

Turney SG, Bridgman PC (2005) Laminin stimulates and guides axonal outgrowth via growth cone myosin II activity. Nat Neurosci 8:717-719.

Vanneste D, Takagi M, Imamoto N, Vernos I (2009) The role of Hklp2 in the stabilization and maintenance of spindle bipolarity. Curr Biol 19:1712-1717.

Walczak CE, Heald R (2008) Mechanisms of mitotic spindle assembly and function. Int Rev Cytol 265:111-158.

Wang L, Brown A (2002) Rapid movement of microtubules in axons. Curr Biol 12:1496-1501.

Wittmann T, Boleti H, Antony C, Karsenti E, Vernos I (1998) Localization of the kinesin-like protein Xklp2 to spindle poles requires a leucine zipper, a microtubule-associated protein, and dynein. J Cell Biol 143:673-685.

Xu T, Qu Z, Yang X, Qin X, Xiong J, Wang Y, Ren D, Liu G (2009) A cotton kinesin $\mathrm{GhKCH} 2$ interacts with both microtubules and microfilaments. Biochem J 421:171-180.

Yoon SY, Choi JE, Huh JW, Hwang O, Lee HS, Hong HN, Kim D (2005) Monastrol, a selective inhibitor of the mitotic kinesin Eg5, induces a distinctive growth profile of dendrites and axons in primary cortical neuron cultures. Cell Motil Cytoskeleton 60:181-190.

Yu W, Ling C, Baas PW (2001) Microtubule reconfiguration during axogenesis. J Neurocytol 30:861-875. 